\title{
Strategies for the Interactive Resolution of Calculus Problems
}

\author{
Fernando Díez and Roberto Moriyón \\ Department of Computer Engineering, Universidad Autónoma de Madrid, \\ 28049 Madrid, Spain \\ \{Fernando.Diez, Roberto.Moriyon\}@ii.uam.es
}

\begin{abstract}
In this work we show how MathEdu, an authoring tool for the design of collections of interactive problems of Mathematics that involve symbolic computation allows the use of resolution strategies. We also describe the mechanisms related to these strategies. MathEdu allows for the definition of interactive problems and their generalization. MathEdu allows also for the definition of resolution strategies and for the characterization of the cases when they can be applied. This process is based on the pattern matching of mathematical expressions. Specific problems are generated in MathEdu from generalized problem patterns by random generation, and students can solve them by means of an interactive dialog with the system, that validates the strategy used by the student and the results of the accomplished tasks.
\end{abstract}

\section{Introduction}

In spite of the considerable advances in Human-Computer Interaction at the end of last century, there is a notorious lack of interactive applications oriented towards the learning of scientific disciplines, and particularly Mathematics. Among the most advanced systems of this kind we can mention the following ones: Cognitive Tutor, [9], is a software implementation of a mathematical curriculum that monitors the process of problem solving by students and adapts itself to their needs. Calculus Machina, [8], is a tool that uses a set of algorithms in order to solve a wide variety of calculus problems and explains the solution in considerable detail. It also allows students to take an active role in the learning process. Finally, EGrade, [6], is webbased software that automates the assignment, delivering, grading, and guidance for homework, quizzes, and exams in scientific subjects. The main drawback of the existing applications of this type is that they are not adapted to the structural complexity of the knowledge to be learned, including the different strategies and methods of problem resolution. We shall also point out that the advances by the Artificial Intelligence community in the development of mechanisms for automated theorem proving and problem solving, [1], [10], have not been used yet to give Mathematics tutoring systems more ability to help students in their work.

For example, most tutoring applications that have been developed up to now usually allow the student to interact with the system either by selecting by means of buttons the right answers to the questions posed among a predefined set of them or, in more sophisticated systems, by typing the answers directly. In any case, the system usually 
does not admit several steps in the resolution of problems, and does not adapt itself in any way to the information supplied by the student.

In this work, starting from the previous considerations, we describe some relevant aspects of MathEdu, [4], [5], an authoring tool for the design of courses based on collections of interactive problems of Mathematics that involve symbolic computation.

MathEdu is implemented on top of Mathematica [1, [11], a system for symbolic computation that has very powerful pattern matching capabilities. This, together with its ability to build mathematical symbolic expressions dynamically, makes Mathematica a particularly suitable tool for the representation and manipulation needed in the kind of problems we address in this work, as we explain with more detail in the remaining sections of this work.

MathEdu includes three modules: MathDesigner allows the definition of sets of problems; MathSolver allows students the interactive resolution of problems, and MathTrainer teaches students how to solve exercises by solving them step by step with detailed explanations.

With MathDesigner, teachers can write down problem statements that can be later generalized by substitution of some parts of the mathematical formulae that appear in them by any other formula that is appropriate for the same type of problem. Just like different problems of the same type are solved in a traditional setting some times similarly, but some other times they are solved by means of different strategies, [7], teachers can also specify by means of MathDesigner strategies that can be used to solve different cases of the same general type of problem. The specification of problem solving strategies by teachers in MathDesigner includes also the definition of a dialog between the student and the system to take place during the resolution of the problem.

MathSolver generates randomly specific problems from the generalized problem patterns defined by the teacher. The resolution of the problems is done by means of an interactive dialog with the system, that validates the strategy chosen by the student. In this dialog MathSolver acts as an interpreter of the tasks defined by the teacher, and takes into account at each step the specifications of mathematical formulae by the student as an answer to the questions posed by the system. This mechanism allows the student to communicate with the system at a level of abstraction that is very similar to the one used by persons in written mathematical texts.

Finally, the exercises solved by MathTrainer can be stated either by the system, by a teacher or by the student; they must correspond to one of the types defined by the teacher.

The combined use of MathDesigner together with MathTrainer and MathSolver allows the design of courses that include both explanations and practice. This is done by intertwining explanatory static texts with dynamical problem resolutions.

In this paper we shall describe the mechanisms that allow MathEdu to include and manage strategies for the resolution of problems. The descriptions of the paper are based on a representative example of an exercise in Integral Calculus. In next section we introduce this example and give a first description of the main features of $M a t h E d u$ at the level of abstraction an advanced user would use to describe it. The following section includes a description of the way the mathematical knowledge used

Mathematica is a registered trademark of Wolfram Research, Inc. 
by $M a t h E d u$ is represented together with some details about its treatment. Finally, the last section includes conclusions and plans for future work.

\section{Resolution Strategies in MathEdu}

In this section we shall describe the mechanisms that lie behind the use of resolution strategies in MathEdu. We shall do it by means of a simple example, which is sufficiently representative as to show in a clear context all the main aspects that we want to point out, including the relevant requirements that MathEdu satisfies. When a student starts solving a problem interactively with MathSolver, the system shows him/her the statement in a window where all the steps in the resolution will be included progressively, together with a palette where one of the strategies that can be used for the kind of problem to solve can be selected. Figure 1 shows a specific example of this kind that corresponds to a problem that consists in computing an indefinite integral. MathEdu does not limit in principle the field of Mathematics or Physics where it can be used, except for the fact that the resolution of problems must consist of the application of manipulations to mathematical formulae. Other possible types of problems are differentiation, computation of limits of functions, resolution of ordinary differential equations, etc.

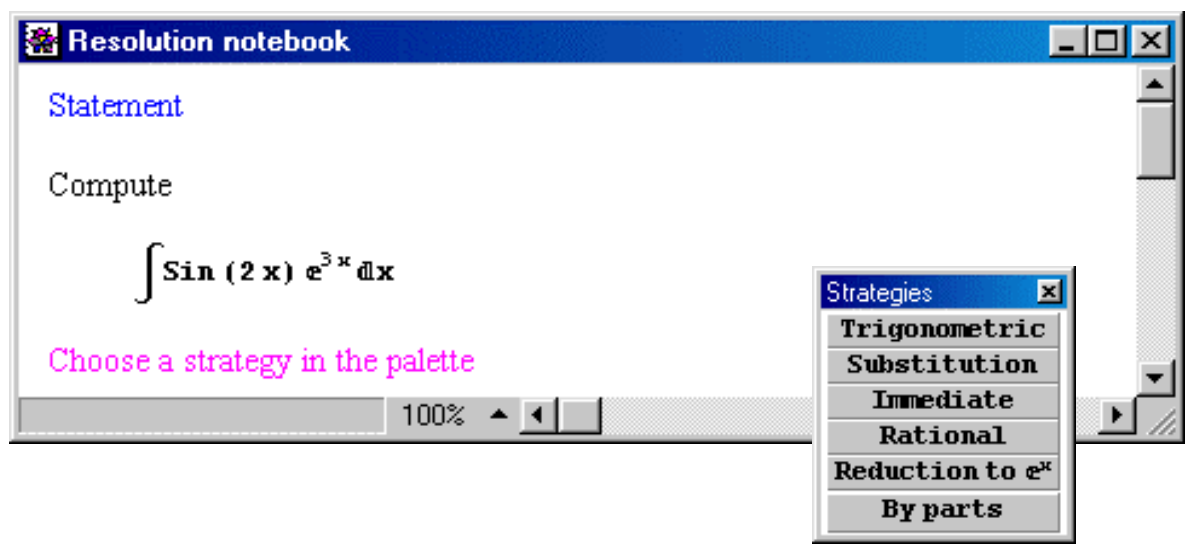

Fig. 1. Statement and palette for strategy selection

In this case there are two strategies that can be used to solve the problem: either by integration by parts or by reducing it through substitution to the integral of a function of $\mathrm{e}^{\mathrm{x}}$. It is worth mentioning that MathSolver is able to notice directly from the statement that in this particular case both strategies can be applied, so it will accept the choice by the student of any of them. In case the student chooses a strategy that can not be applied, the system will show a message of explanation that will include a description of the conditions that must be satisfied for the chosen strategy being acceptable. This default behavior can be changed by the teacher.

$M a t h E d u$ also allows the teacher to give the student the possibility to do optionals manipulations to the data of the problem, like algebraic simplifications. For example, 
if the student is asked by the system to compute the derivative of $(x+1) /\left(x^{2}-1\right)$, the palette for strategy selection can include an option called Make previous computations. When choosing this, the student can transform the rational fraction to irreducible form. She/he can also also simplify the result of the problem. In case the work of the student is being graded by the system, his/her rating will be higher if simplifications are used.

Before going into the description of the mechanisms behind the management of resolution strategies we will address some previous questions. The first one is related to the possibility that there are different cases where the same strategy can be used. This is obvious in our example, since integrals that can be solved by parts must have an integrand that is the product of two expressions, according to one of several possible patterns. The first three possibilities correspond to one of the factors being a polynomial and the other being either an exponential, a logarithm or a simple trigonometrical function (sine or cosine). In the fourth case the integrand is the product of a simple trigonometrical function by an exponential, and in the fifth one it consists of the product of two simple trigonometrical functions whose arguments are multiplied by different coefficients. The example in figure 1 corresponds to the fourth case. Once again, given any problem that corresponds to one of these five cases MathSolver is able to notice that it can be solved by means of integration by parts. Only in the fourth case it will accept the strategy of reduction to a function of $e^{x}$. However, the system does not need any explicit information of this kind, since the teacher who designs a course just has to specify the conditions on the integrand on which each resolution method can be applied.

Another question to take into account is the way in which the problem is posed. In principle this can be done either by the system or by a user (student or teacher). In the last case it is the responsibility of the user to pose a problem that can be solved with the knowledge that has been incorporated into MathEdu. In the first one, a random mechanism is used that will be explained next.

First of all, a type of problem must be given. In case the student is following a course, the type of problem is usually chosen by the system. In other cases it can be chosen by the tutor or by the student. It can also happen that the purpose of the session is to check how the student solves the problem that is posed to him/her by a specific method or methods, or that the use of any strategy that is suitable will be accepted. We shall assume that we are in this case, the other ones presenting minor changes with respect to it. In the same way, a strategy and a case must be chosen; similar questions to the ones commented above must be taken into account for this. Let us assume that the final decision is to pose a problem that corresponds to the fourth case among the ones that can be solved by the strategy of integration by parts. The description of these problems used by MathEdu is that they must ask for the computation of an indefinite integral of the form

$$
\int u(x) \cdot V(x) d x
$$

where $u(x)$ must be a simple trigonometric function and $V(x)$ must be an exponential function. The first condition (the decomposition as a product) is imposed in all the cases that correspond to the strategy of integration by parts. The integral that appears in (1) constitutes a generalization of the one in the statement shown in figure 1. 
In general, abstract problems that correspond to a strategy are represented by statements whose formulae include structural patterns as in (1). The parts of those structural patterns whose values can change from one specific problem to another, $u$ and $V$ in our example, are called metavariables. As a part of the design of a set of interactive problems, the teacher writes down specific problems like the one in figure 1 , and then generalizes them by the definition of associated metavariables. This process is done interactively in the same context where the problems that correspond to the generalization will be solved, using Programming by Demonstration techniques [2].

We have already seen that the metavariables that appear in our example must satisfy some conditions. In general, for each case that corresponds to a given strategy, all the expressions that can substitute a metavariable in specific problems that correspond to that case must satisfy a specific condition. This condition can consist in the expression being a polynomial, an exponential, a simple trigonometric function, etc. Both the structural patterns that correspond to each strategy, as the one in (1), and the conditions associated to metavariables and cases are specified by the teacher when designing the course or set of problems. This specification is not too complex, since it involves natural mathematical operators.

On the other hand, MathSolver generates problems from abstract problem types by means of random generation of mathematical expressions. This process has two interesting features: First, the expressions that are randomly generated are not the whole formulae that appear in the statement, but some parts of them, that appear as metavariables in the abstract problem. Moreover, the random expressions can have a specific structure. In our example, MathSolver would have to chose a random expression of the form $\sin (K x)$ or $\cos (K x)$ for $u(x)$ and another random expression of the form $\mathrm{e}^{\mathrm{Cx}}$ for $V(x)$. In other examples, the generation of random polynomials or even the random generation of functions of the form $f(g(x))$ is needed, as when a statement of a problem that can be solved by change of variables is wanted. Hence, besides conditions, each case and each metavariable have also an associated random generator of expressions that substitutes the metavariable in the corresponding pattern when generating specific problems for the desired abstract case problem.

We shall describe now the mechanisms involved when verifying the correctness of the selection of a strategy by the student for the resolution of a given problem. Although problems are generated according to a particular case for a given strategy and problem type, we have already seen that there is the possibility that other strategies can be used for their resolution. For example, in the case of the problem stated in figure 1 the student can choose the use of integration by parts if the problem has been generated from this strategy, but also if it has been generated from the strategy of reduction to the computation of an integral involving only exponentials. Hence, the system must verify directly from the type of problem, the specific formulae that appear in its statement, and the strategy selected by the student, whether the chosen strategy can be used or not in the resolution of the problem.

This verification can be done as follows: for each problem case that is associated to the strategy of the student, the system tries to match all the formulae in the statement of the problem with the complete conditional pattern that corresponds to the case, that takes into account the conditions imposed on the metavariables. In our example, the formula 


$$
\int \operatorname{Sin}(2 x) \cdot e^{3 x} d x
$$

is matched against the conditional pattern

$$
\int u_{-} ? \operatorname{SimpleTrig} Q \cdot V_{\_} ? \operatorname{Exp} Q d x
$$

together with four other ones, giving in this case a positive answer with the substitution

$$
\begin{aligned}
& u \rightarrow \sin (2 x) \\
& V \rightarrow e^{3 x}
\end{aligned}
$$

The adaptation of the system we have just described, accepting resolution methods proposed by the student different from the one that was originally planned, has no precedents in other tutoring systems.

Once the choice of a strategy made by the student has been validated, the system starts a dialog with the student based on the interpretation and interactive execution of the sequence of tasks attached to the selected strategy. For example, when solving the problem shown in figure 1, the system will ask the student about the next steps to be done, including among several possibilities the determination of the values of $u$ and $\mathrm{V}$. There is a library of predefined tasks and dialog fragments that can be used by the designers. These include choices to be made by the student, input of mathematical functions by the student and the resolution of simpler subproblems whose results can be used in the resolution of the previous problem. For example, the computation of an integral by parts involves the resolution of two simpler integrals: the integral of $\mathrm{V}$, that usually is an immediate one, and another one obtained from the result of this previous subproblem and by differentiating the metavariable $u$. Similarly, the computation of the integral of a rational function like

$$
\int \frac{3 x^{2}+1}{x^{3}+x} d x
$$

can be done through a change of variables that leads to the resolution of a simpler integral, and the solution of this subproblem is used in order to find the solution to the original one. Allowing the resolution of problems in terms of simple steps and the resolution of subproblems is an essential aspect of MathEdu functional design.

Mathematica, with its extraordinary capability of symbolic manipulation and its powerful pattern matching system, is especially suitable for the kind of work described up to now. This includes the dynamical execution at a symbolic level of complex mathematical computations on formulae that are not known at the instant the system is developed, in a way similar to the manipulation of mathematical formulae by persons, and the determination of deep structural properties of mathematical expressions, like the ones mentioned in the previous paragraphs. Conditions and generators that are needed in the design process are included in a library that is interpreted at execution time. They can reduce considerably the amount of work during the design of a course that involves a set of problems. However, together with these features, Mathematica is especially limited from the point of view of the kind of 
interaction allowed to the user with the system and the way this interaction can be designed and programmed.

\section{Representation of Mathematical Knowledge in MathEdu}

The knowledge needed to accomplish the tasks described in the previous section involves standard knowledge representation plus specific knowledge related to mathematical formulae, their manipulation and pattern matching. The fundamental blocks that conform this knowledge are mathematical expressions (MEs), ME patterns and ME manipulators, which transform them by means of the usual symbolic computations people do when working on Mathematics. ME patterns have a similar structure to MEs, and can include references to metavariables. Hence, a formula like the one in (1) can be represented by means of a ME pattern in a context in which both $\mathrm{u}$ and $\mathrm{V}$ are metavariables. A mathematical text is a sequence of plain text and $\mathrm{ME}$ patterns. Metavariables can be represented by means of a condition that is an ME pattern and a generator that is an ME manipulator. Problem cases, a more complex concept, consist essentially of a generic problem statement (a mathematical text) and a sequence of metavariables. Dialog components define tasks that are part of a resolution strategy and depend on the type of the component and messages to be used in the dialog. Strategies are formed by the associated ME patterns, problem cases and a sequence of interactive tasks. And finally, the main concept used in the design of $M a t h E d u$ is that of a Problem Type, that consists of a generic statement, and a set of strategies.

With respect to the course structure, a course unit is formed by a problem case which leads to a specific problem to be solved by the system step by step, including explanations to the student about its resolution, corresponding mathematical texts that serve as introduction and afterwards explanations, and other specific problems to be solved interactively by the student. Next, a paragraph consists of a strategy, an introductory mathematical text, a sequence of units with problem cases that correspond to that strategy, and a set of problems that correspond to the different problem cases. Finally, a chapter consists of a problem type, an introductory mathematical text, a series of paragraphs whose strategies correspond to the problem type, and a sequence of specific problems to be solved.

The representation of all the previous concepts by data structures is done in MathEdu by means of the Mathematica system, that allows symbolic structures to be interleaved with the more standard ones, and whose strong symbolic capabilities are specially suited for this integration.

Now we are prepared to point out some of the aspects related to the mechanisms explained in the previous sections that need additional clarification. Figure 2 shows a partial textual representation of the data structures associated to the type of problem that corresponds to the one in figure 1, which consists in the computation of an indefinite integral. We shall start by the description of the process of random exercise generation. Figure 2 shows the order in which the different steps involved are taken. We assume that the selected problem type and strategy are Integration and ByParts. At this point in the generation process, the deep structure of the statement and the resolution tasks of the exercise are univocally determined. Now, the statement must be built. This is done in four steps. In the first one, the symbols of the metavariables 
are selected. Next (step 2), the system activates the generators that correspond to each metavariable. These generators build the corresponding mathematical structures (step3), which are propagated and substituted in the corresponding formulae patterns and finally in all the formulae in the statement (step 4).

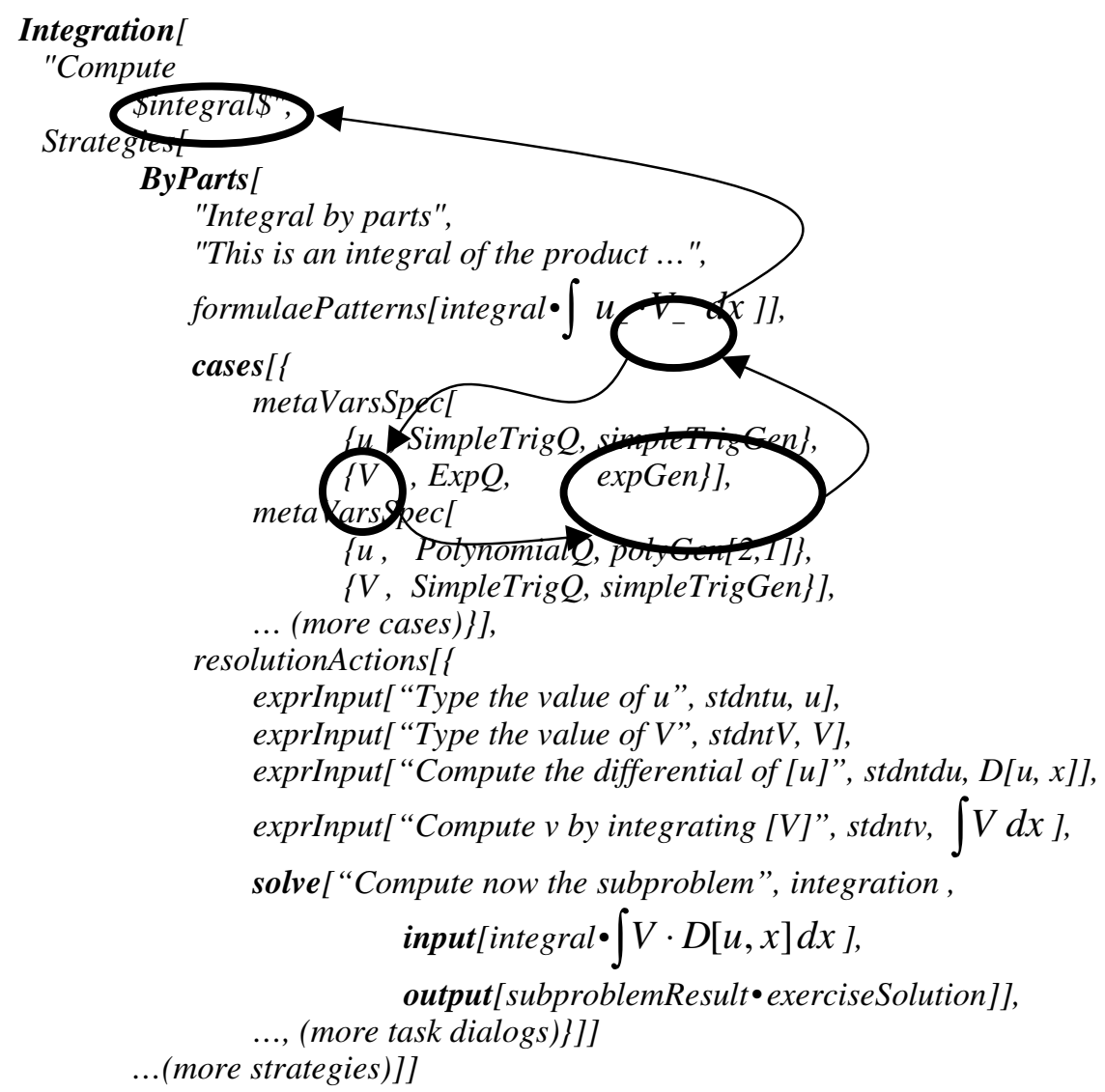

Fig. 2. Use of knowledge representation in MathEdu: Problem type

In what follows we shall describe the validation of the strategy proposed by the student during the interactive resolution of a problem. The critical point is the construction of the full pattern whose satisfaction must be checked for every formula and each problem case. This pattern is formed as the conjunction of the ME pattern associated to the strategy ( $\int u_{-} \cdot V_{-} d x$ in figure 2 ), and the different conditional patterns associated to the problem case under study (SimpleTrig $Q$ and $\operatorname{Exp} Q$ in the first case of figure 2), that correspond to all the metavariables. In particular, in our example we get the pattern (2). The comparison with this pattern is equivalent to the successive comparison with all the patterns that correspond to the metavariables under the problem case being analyzed. 
Finally, Figure 2 also shows the structures that correspond to resolution actions. We shall just point out the solve action, that gives rise to a recursive call to the MathSolver or MathTrainer main routine in order to solve a subproblem. The solve action specifies a specific problem to be solved by means of a problem type and the specific values for its metavariables (input) in terms of the data available in the resolution of the original problem. It also indicates how the result of the subproblem is incorporated to metavariables of the original problem (output).

The statement in figure 1 is one of the possible results of this process. Once the statement has been generated and shown to the student in the resolution notebook, the interactive resolution can start according to the explanations in the previous section.

\section{Conclusions}

In this work we have seen how pattern matching of mathematical formulae allows the automatic determination of the resolution strategies that can be applied to problems of Mathematics that involve symbolic computation. We have also seen how the same techniques are useful for the automatic generation of problems where a particular strategy can be applied. Moreover, when more that one strategy can be used for the resolution of a problem, this allows a computer based tutoring system to adapt itself to the strategy selected for a student in case it is valid. The MathEdu system includes these capabilities.

In the near future we are planning to add more functionality to MathEdu by means of the inclusion of a model of the student that will allow the system to determine the capabilities and skills of each application user. This will allow the presentation of exercises adapted to the skills of the student. Therefore the student will see the tutoring system as a collaborator.

We shall continue working on the deep structure of mathematical objects, their representations and their interactions [3], in order to extend the fields of application of MathEdu.

Finally, we are also conducting work intended to the improvement of MathEdu in order to allow the possibility to use it through Internet.

Acknowledgements. This work has been funded by the Plan Nacional de I+D of Spain, project TEL1999-0181.

\section{References}

1. Castells, P., Moriyón, R., Saiz, F.: Solving Mathematical Problems that Involve Reasoning and Calculations. In Proc. Golden West IV International Conference on Intelligent Systems. S. Louis, ed. International Society for Computers and Their Applications, USA (1995)

2. Cypher, A.: Watch what I do. Programming by Demonstration. The MIT Press, Cambridge, MA, USA (1993) 
3. Díez, F., García, M.C.: Supervisión automática del aprendizaje matemático. Proc. 8th International Congress on Mathematical Education (ICME-8). Sevilla, Spain (1996)

4. Díez, F., Moriyón, R.: Doing Mathematics with MathEdu. IXth Conference of Mathematics/Science Education \& Technology. Association for the Advancement of Computing in Education (AACE) (1999)

5. Díez, F., Moriyón, R.: Teaching Mathematics by Means of MathTrainer. XIIth International Conference of the Society for Information Technology \& Teacher Education. Association for the Advancement of Computing in Education (AACE) (2001)

6. Orr, J.L., Franklin, B.: Egrade: Student Learning Guide. John Wiley and Sons (2000)

7. Pitrat, J. Métacoinnaisance, futur de l'intelligence artificielle. Hermes, Paris (1990)

8. Quinney, D.: Calculus Machina: An intelligent tutor providing computer based support for teaching undergraduate calculus. Proc. 2nd Int. Conference on the Teaching of Mathematics. Hersonissos, Crete (2002)

9. Ritter, S., Koedinger, K. R.: An architecture for plug-in tutor agents. Journal of Artificial Intelligence in Education, 7 (1996) 315-347.

10. Voronkov, A., ed.: Proc. CADE-18. Lecture Notes in Computer Science. Springer Verlag (2002)

11. Wolfram, S.: The Mathematica Book, 4th Edition. Cambridge University Press (1999) 